\title{
Factores Asociados Con La Falta De Éxito En La Implementación De La Planeación Estratégica (IPE) En Empresarios Y Directivos Del Centro Empresarial De Ciudad Juárez, Chihuahua, México 2014-2015
}

\author{
Edgar Lara Enríquez, MI \\ Jesús Alberto Urrutia de la Garza, PhD \\ Carlos Jesús González Macías, PhD \\ Universidad Autónoma de Ciudad Juárez, Mexico
}

URL:http://dx.doi.org/10.19044/esj.2020.v16n1p23

\section{Resumen}

El ejercicio de la Planeación Estratégica (PE) en las organizaciones es fundamental en la toma de decisiones para la elección de mejores alternativas y asignación óptima de recursos para alcanzar una visión de futuro. Las readecuaciones de la PE se originan entre su diseño y su implementación, por lo que es importante analizar la brecha formada entre donde culmina la planeación y cuando comienza a implementarse. La Implementación de la Planeación Estratégica (IPE) está asociada a diversos factores que generan el éxito o fracaso para el cumplimiento de la visión de las organizaciones empresariales. Los factores cambian en función del contexto en el proceso de la IPE. El desarrollo del trabajo es identificar los factores que contribuyen al éxito o fracaso de la IPE para establecer un modelo de readecuación de la Planeación Estratégica. En este trabajo se encuestaron a 85 empresarios y/o directivos participantes directos e indirectos del Centro Empresarial COPARMEX Juárez, México. En el grupo de estudio se obtuvieron hallazgos que determinan que las variables: visión compartida, liderazgo y comunicación están asociadas con la falta de éxito o fracaso de la PE.

Palabras clave: Planeación Estratégica, Factores de éxito, Empresarios, Centros empresariales, Ciudad Juárez 


\title{
Associated Factors to the Lack of Success of Strategic Planning Establishment (SPE) in Businessmen and Directors from the Entrepreneurial Center in Ciudad Juarez, Mexico, 2014-2015
}

\author{
Edgar Lara Enríquez, MI \\ Jesús Alberto Urrutia de la Garza, PhD \\ Carlos Jesús González Macías, PhD \\ Universidad Autónoma de Ciudad Juárez, Mexico
}

\begin{abstract}
The exercise of Strategic Planning (SP) in organizations is essential for decision making in order to choose better alternatives and optimal resource display to achieve a future-focus vision. Readjustments in SP are originated between its design and its implementation, therefore is important to analyze the formed gap between where planning ends and where implementation begins. The Establishment of Strategic Planning (ESP) is associated to many factors that causes success or failure in fulfilling the vision of business organizations. Those factors change in relation of the ESP process context. The development of this study is to identify those factors that contribute to ESP's, in order to establish an SP readjustment model. This research surveyed 85 entrepreneurs and/or directors that direct or indirectly participate with the Entrepreneurial Center COPARMEX Juarez, Mexico. In the study group, findings were obtained that show that variables: shared vision, leadership and communication are associated with the SP's lack of success or failure.
\end{abstract}

Keywords: Strategic Planning, Success factors, Businessmen, Entrepreneurial centers, Ciudad Juarez

\section{Introduction}

La etapa de planeación es crucial dentro del proceso administrativo organizacional, contribuye a la construcción de estrategias integradas para el logro de una visión. Las organizaciones operan en entornos globalizados y competitivos de alto desarrollo tecnológico que las obliga a buscar ventajas competitivas sostenibles mediante la Implementación de Planeación Estratégica (IPE) (Moreno, 2014). 
Los modelos de Planeación Estratégica (PE), observan cierta similitud entre los elementos que los integran. Para conceptualizar y realizar planes de cualquier naturaleza consideran, básicamente, las siguientes etapas: formulación, implementación y evaluación (Guerras, 2004). De acuerdo a lo anterior, este documento se estructuró de la siguiente manera: en primera instancia, se presenta un apartado introductorio, seguido de los antecedentes y el planteamiento del problema; después se incluye la sección de revisión de literatura; así mismo, continúa con una sección propia sobre la metodología utilizada; seguida de una cuarta sección donde se presentan los hallazgos y conclusiones obtenidos del trabajo de investigación. Se presenta al final una sección que contiene las referencias bibliográficas consultadas.

\section{Antecedentes}

La PE se refiere a lograr la alineación de metas y objetivos de una organización racional en función del tiempo específico, pues operan bajo un esquema de competencia e incertidumbre externa, de cambios constantes y competiendo en un mercado específico complejo desarrollando ventajas competitivas enfocadas en la cadena de valor, y adoptada por las empresas que vieron la necesidad de implementar procesos competitivos de largo plazo en mercados inciertos y mejorar su posicionamiento entre competidores directos, indirectos y potenciales, así como clientes y proveedores (Conrado, 2000; Porter, 1980). El presente trabajo plantea el identificar aquellos factores asociados con el éxito o fracaso de la IPE y determinar cómo afrontarlos a través de un proceso de readecuación que permita retomar de manera expedita el proceso de planeación con los ajustes necesarios.

\section{Planteamiento del problema}

El ejercicio de Planeación Estratégica (PE) en las organizaciones es fundamental en la toma de decisiones para la elección de mejores alternativas y asignación óptima de recursos para alcanzar una visión de futuro. La PE establece un procedimiento sistemático para la adecuada previsión de las acciones a desarrollar para la consecución de objetivos, presentando variaciones más relacionadas con la naturaleza y características de la organización que con la metodología o formas de construir sus diferentes etapas, pues constituye uno de los elementos centrales en la toma de decisiones, elección de alternativas y formulación y asignación óptima de recursos económicos.

Dado que el proceso de la IPE comprende varias etapas, pueden existir factores que complican la implementación de los planes, ya sea por factores internos de la organización o factores ajenos a ella (Zaribaf \& Hamid, 2014; Seyed, Reza, Asghar \& Karimy, 2011; Guerras, 2004) originando problemas entre el diseño del plan y su implementación, los cuales pueden ser 
estructurales, como liderazgo, falta de visión compartida, falta de comunicación; o bien externas a la organización, como procesos normativos gubernamentales, aspectos sociales, políticos e incluso económicos (Zaribaf \& Hamid, 2014; Messah, 2011; Deloitte y Stracienta 2007).

Las readecuaciones se originan entre el planteamiento y la implementación, por lo que es importante analizar la brecha formada entre donde culmina la planeación y cuando comienza a implementarse. De acuerdo con Deloitte y Stracienta (2007, p.9), "...al menos el 50\% de las empresas mexicanas con estrategias de negocio padecen deficiencias para ejecutarlas", debido a lo anteriormente descrito, "esa falla en la implementación está originada por diversos factores, algunos de los cuales, han sido determinados por varias investigaciones" (Zaribaf \& Hamid, 2014: Seyed et. al., 2011; Guerras, 2004). No obstante, se desconoce cuáles son los factores que afectan este proceso en un entorno empresarial en Ciudad Juárez, Chihuahua, México. Por lo que se plantea identificar estos factores asociados con el éxito o fracaso de la implementación de la IPE y establecer un modelo de readecuación de la IPE que le permita ser oportuna y expedita en la organización. De acuerdo con lo anterior, se desprende la siguiente pregunta de investigación:

¿Cuáles son los factores asociados con el éxito o fracaso de la IPE que aparecen principalmente entre el planteamiento y su fase de implementación?

Así mismo, se plantea la siguiente hipótesis: Existen factores que dificultan la IPE en las organizaciones empresariales que permita el cumplimiento de las metas de manera oportuna y expedita en un proceso.

\section{Revisión de literatura}

El nuevo institucionalismo parte de la necesidad de la construcción de reglas que están asociadas a la creación de una institución de carácter estructural, es decir; enfocada a los procesos, estrategias y perspectivas de competencia para su desarrollo e interrelación con y entre elementos externos e internos de la misma (De la Rosa, 2002; DiMaggio y Powell, 1991). Es importante distinguir entre institución y organización, la primera tiene carácter del proceso del control del poder a través de reglas. A la organización se le considera un instrumento para el cumplimiento de metas, objetivos y a través del escalamiento de los antes mencionados, las organizaciones se convierten en instituciones y estas últimas pierden su carácter instrumental (Robles, 2014; De la Rosa, 2002).

Las organizaciones modernas tienen la necesidad de realizar planes y alinear sus objetivos a través de una visión para el funcionamiento racional, mediante la identificación de metas como un esquema de armonía en un sistema de consenso de intereses entre el esquema de poder y la visión de la organización, bajo un concepto utilitarista y racionalidad económica (Robles, 2014; De la Rosa, 2002; DiMaggio y Powell, 1991). 


\section{Existe una vasta revisión de las distintas teorías de la organización que desde distintos enfoques han planteado soluciones para las diferentes problemáticas de las empresas. (Ver tabla 1).}

Tabla 1. Principales teorías de la organización

\begin{tabular}{|c|c|c|c|c|}
\hline No. & Teoría & $\begin{array}{l}\text { Periodo } \\
\text { (Tiempo) }\end{array}$ & $\begin{array}{l}\text { Principal } \\
\text { Autor }\end{array}$ & $\begin{array}{l}\text { Principales aportaciones } \\
\text { teóricas, }\end{array}$ \\
\hline 1 & Científica & 1900 & $\begin{array}{l}\text { Frederik Taylor } \\
1900\end{array}$ & $\begin{array}{l}\text { La mejor forma de organización es la } \\
\text { que permite medir el esfuerzo } \\
\text { individual. }\end{array}$ \\
\hline 2 & Funcional & 1916 & $\begin{array}{l}\text { Henry } \\
1916\end{array}$ & $\begin{array}{l}\text { La mejor forma de organización es la } \\
\text { que está basada en una división de } \\
\text { funciones, que dividen en subfunciones } \\
\text { y procedimientos. }\end{array}$ \\
\hline 3 & Burocrática & 1924 & $\begin{array}{l}\text { Max } \\
1924\end{array}$ & $\begin{array}{l}\text { La mejor forma de organización es la } \\
\text { que tiene reglas claras y racionales, } \\
\text { decisiones impersonales y excelencia } \\
\text { técnica de sus empleados y gestores. }\end{array}$ \\
\hline 4 & Relaciones Humanas & $1920-1957$ & $\begin{array}{l}\text { Mayo, 1920; } \\
\text { Follet, Maslow, } \\
\text { 1954; Herzberg, } \\
\text { 1966; } \\
\text { McGregor, } 1957\end{array}$ & $\begin{array}{l}\text { La mejor forma de organización es la } \\
\text { que considera e integra a las personas } \\
\text { que la hacen funcionar. }\end{array}$ \\
\hline 5 & Sistemas cooperativos & 1938 & $\begin{array}{l}\text { Chester } \\
\text { Bernard, } 1938\end{array}$ & $\begin{array}{l}\text { La mejor forma de organización es la } \\
\text { que asegura la cooperación de los } \\
\text { miembros que conforman la } \\
\text { organización mediante un trato justo y } \\
\text { beneficios recíprocos. }\end{array}$ \\
\hline 6 & General de Sistemas & $1930-1947$ & $\begin{array}{l}\text { Beterlanffy, } \\
\text { Katz, } \\
\text { Rosengweiz }\end{array}$ & $\begin{array}{l}\text { La mejor forma de organización es la } \\
\text { que coordina armónicamente los } \\
\text { diferentes subsistemas que definen el } \\
\text { sistema organizacional. }\end{array}$ \\
\hline 7 & Comportamiento & 1961-1984 & $\begin{array}{l}\text { Herbert Simon, } \\
1961\end{array}$ & $\begin{array}{l}\text { La mejor forma de organización es la } \\
\text { que permite que los empleados de } \\
\text { todos los niveles tomen decisiones y } \\
\text { colaboren en el cumplimiento de los } \\
\text { objetivos de acuerdo a su nivel de } \\
\text { influencia y autoridad. }\end{array}$ \\
\hline 8 & Contingencia & 1972 & $\begin{array}{l}\text { Burns, Slater, } \\
\text { Woodward, } \\
\text { Child }\end{array}$ & $\begin{array}{l}\text { La mejor forma de organización } \\
\text { depende de su tecnología tamaño y } \\
\text { medio ambiente. }\end{array}$ \\
\hline 9 & Política & $1957-1960$ & $\begin{array}{l}\text { Selznick, } \\
\text { Pfeffer, Croazier }\end{array}$ & $\begin{array}{l}\text { La mejor forma de organización es la } \\
\text { que crea colaboraciones entre los } \\
\text { diferentes grupos de interés de la } \\
\text { organización y gestiona de manera } \\
\text { positiva el conflicto. }\end{array}$ \\
\hline 10 & $\begin{array}{l}\text { Desarrollo } \\
\text { Organizacional }\end{array}$ & 1946 & $\begin{array}{l}\text { Kurt } \\
1946\end{array}$ & $\begin{array}{l}\text { La mejor forma de organización es la } \\
\text { que promueve el cambio planeado } \\
\text { basado en intervenciones donde la } \\
\text { colaboración entre distintos niveles } \\
\text { organizacionales es posible. }\end{array}$ \\
\hline 11 & Institucional & $1977-1983$ & $\begin{array}{l}\text { DiMaggio, } \\
\text { Powell, } 1983\end{array}$ & $\begin{array}{l}\text { La mejor forma de organización es la } \\
\text { que considera e integra a las personas } \\
\text { que la hacen funcionar }\end{array}$ \\
\hline
\end{tabular}


12

Recursos

capacidades

13

Agencia

Sistemas alejados el equilibrio

Criticabilidad

organizada у 1991

1991

1977- 1984

1975-1985

1993

1980-1984

1980
Rummel,

Schendel,

Teece, 1991

Barney, 1991

Hannan, 1977;

Freeman, 1984

Williamson, 1975, 1985

Cambell, 1993

Llya Prigione, 1980

Maturana,

Varel, 1980
La mejor forma de organización es la que gestiona más racionalmente sus recursos y capacidades.

Es la que crea mecanismos que previenen que actores actúan a favor de sus propios intereses y lo premia si actúa a favor de los intereses de la organización.

La mejor forma de organización es la que consigue adaptarse al entorno y seguir operando con eficiencia.

La mejor forma de organización es la que se enfoca a la minimización de los costos de transacción.

Es la que gestiona la variabilidad caótica de la organización gestionando su complejidad.

Es la que permite adaptarse y auto organizarse.

Crea una red de procesos u operaciones y que pueden crear o destruir elementos del mismo sistema.

Fuente: Elaboración propia en base a Rivas, 2007.

Las corrientes teóricas organizacionales relacionadas con la IPE son la escuela del comportamiento y la teoría de la contingencia. (Okari, Letting, Gachunga, Katula, 2013; De la Rosa, 2002; Ramírez et. al. 2001). El enfoque de la contingencia empresarial representa el desarrollo de la planeación en las organizaciones empresariales, ubicando a la empresa como un sistema abierto donde los esquemas racionalistas dan pie a la necesidad de conformar modelos y estructuras organizacionales minimizando riesgos y aumentando el éxxito en la implementación de la planeación (Rivas, 2007).

Una de sus aportaciones más interesantes es el análisis del poder de las organizaciones como un factor clave instrumental para el logro de objetivos como estrategia corporativa, considerando como falla el uso inadecuado del poder de jerarquía en la implementación de la estrategia empresarial (Chinchilla, 1991).

De acuerdo a lo anteriormente expuesto, este trabajo resulta pertinente dado que el objetivo es identificar los factores que contribuyen al éxito o fracaso en una IPE oportuna y expedita, para el logro de consensos empresariales entre diversos actores claves, concretamente en la Confederación Patronal de la República Mexicana (COPARMEX) del Centro Empresarial de Ciudad Juárez, México, debido a la influencia que representa en el impacto económico y social en la región. 


\section{Metodología}

Se analizaron los factores que afectan la Implementación de la Planeación Estratégica (IPE), para ello fue necesario determinar las dimensiones en donde se sustenta la evidencia empírica para la pertinencia de las preguntas y/o variables que se seleccionan para el análisis de datos. (Ver tabla 2). Las debilidades más sobresalientes en los modelos de Planeación Estratégica (PE) detectadas son: Visión, Liderazgo, Comunicación efectiva.

Tabla 2. Fortalezas y debilidades de los Modelos de Planeación

\begin{tabular}{|c|c|c|c|c|c|c|c|}
\hline \multirow[b]{2}{*}{ No. } & \multirow[b]{2}{*}{ Modelo } & \multicolumn{3}{|c|}{ Fortalezas } & \multicolumn{2}{|c|}{ Debilidades } & \multirow[b]{2}{*}{$\begin{array}{c}\text { Identificaci- } \\
\text { ón de } \\
\text { liderazgos }\end{array}$} \\
\hline & & $\begin{array}{l}\text { Procesos } \\
\text { internos }\end{array}$ & $\begin{array}{c}\text { Diagnó- } \\
\text { stico }\end{array}$ & $\begin{array}{c}\text { Perspecti- } \\
\text { va de } \\
\text { largo } \\
\text { plazo }\end{array}$ & $\begin{array}{l}\text { Socializa- } \\
\text { ción }\end{array}$ & $\begin{array}{c}\text { Visión } \\
\text { de } \\
\text { futuro }\end{array}$ & \\
\hline 1 & $\begin{array}{l}\text { William } \\
\text { Newman }\end{array}$ & & $X$ & & & $\mathrm{X}$ & \\
\hline 2 & $\begin{array}{l}\text { Frank } \\
\text { Banghart }\end{array}$ & $X$ & & $X$ & $X$ & & \\
\hline 3 & Tom Lambert & $\mathrm{X}$ & & $\mathrm{X}$ & $\mathrm{X}$ & & \\
\hline 4 & $\begin{array}{ll}\text { Colón } & y \\
\text { Rodríguez } & \end{array}$ & $X$ & & & $X$ & $\mathrm{X}$ & $\mathrm{X}$ \\
\hline 5 & $\begin{array}{l}\text { Bateman } \quad y \\
\text { Snell }\end{array}$ & $\mathrm{X}$ & & $\mathrm{X}$ & $\mathrm{X}$ & $\mathrm{X}$ & \\
\hline 6 & $B I D$ & $X$ & $X$ & & $X$ & & $X$ \\
\hline 7 & $S H K G$ & $\mathrm{X}$ & & & & & $\mathrm{X}$ \\
\hline 8 & $\begin{array}{l}\text { Statos, Etzel } \\
y \text { Walter }\end{array}$ & $X$ & & & $X$ & $\mathrm{X}$ & $X$ \\
\hline 9 & Raph Mroz & $\mathrm{X}$ & $\mathrm{X}$ & & & & $\mathrm{X}$ \\
\hline 10 & Kaufman & $X$ & & $\mathrm{X}$ & $X$ & & $X$ \\
\hline
\end{tabular}

Fuente: Elaboración propia

Se determinó una línea de enfoque de estudio a través de las dimensiones determinadas en los modelos de planeación: Visión. Los factores de esta dimensión tienen que ver con la visión compartida institucional y directiva para el éxito y/o fracaso en la IPE (Zaribaf \& Hamid, 2014; Estrada, García \& Sánchez, 2009; Guerras, 2004). En la dimensión de la Visión se determinaron cinco vertientes (visión correcta, construcción de la visión, importancia de la visión compartida, visión retadora, peso de la visión) que muestra la evidencia empírica y que se utilizan de sustento para definir las preguntas genéricas. Liderazgo. Las dimensiones identificadas tienen el enfoque de las organizaciones empresariales, el liderazgo juega un papel altamente relevante en la implementación de la Planeación Estratégica (Zaribaf \& Hamid, 2014; Hasan Pour, 2011; Seyed, Reza, Asghar \& Karimy, 2001; Guerras, 2004). Para el caso de la dimensión de Liderazgo se determinan seis elementos (factor clave del liderazgo, características del líder, rol del líder, delegación de implementación, paradigmas, peso del liderazgo, habilidades 
del liderazgo) que se agrupan en cuatro preguntas. Comunicación efectiva. Se concreta en la comunicación de la visión en todas las jerarquías de la organización interna y externa para el éxito o fracaso de la IPE. (Zaribaf \& Hamid, 2014; Hasan Pour, 2011; Messah, 2011; Guerras, 2004). Para el caso de la Comunicación efectiva se identificaron ocho vertientes (facilitación de la comunicación, integración de actores, formas de comunicación de la visión, tipo de comunicación externa o interna, comunicación formal o informal, permanencia de la comunicación, cultura de comunicación, peso de la comunicación) que fueron condensadas en cinco. Se consideraron otros factores que afectan la IPE (influencia de otros factores, asignación de recursos, estructura organizacional, sistemas internos de control, recompensas por objetivos, otros). Criterio de inclusión. Para determinar el marco muestral, se partió del listado de participantes directos e indirectos del Centro Empresarial COPARMEX Juárez que asciende a 108 empresarios o directivos con las características siguientes: 1.- Empresario o directivo participante en el Centro Empresarial COPARMEX Juárez. 2.- Experiencia en los procesos de planeación interna de su compañía. 3.- Participante activo en los procesos de planeación del Centro Empresarial COPARMEX Juárez. No se determinó ningún criterio de exclusión. Con el fin de obtener una muestra representativa de individuos elegibles, se utilizó el método aleatorio simple para seleccionar la unidad de muestra del listado de participantes. Para determinar el muestreo probabilístico, se procedió con un margen de error del 5\% y un nivel de confianza del $95 \%$. En base a lo anterior y utilizando la fórmula de población finita, se determinó la muestra de 85 encuestas.

Construcción del cuestionario. La necesidad de obtener datos relevantes para el cumplimiento de los objetivos propicia la pertinencia de su recopilación, para ello se parte de la evidencia empírica de los factores identificados que dificultan la IPE. (Ver tabla 3).

Tabla 3. Factores identificados que dificultan la implementación

\begin{tabular}{|c|c|c|c|c|c|c|c|}
\hline No. & Factores & $\begin{array}{c}\text { ZARIB } \\
\text { AF, } \\
\text { HAMI } \\
\text { D } 2014\end{array}$ & $\begin{array}{c}\text { HAS } \\
\text { AN } \\
\text { POU } \\
\text { R } \\
2011\end{array}$ & $\begin{array}{c}\text { SAYED,REZ } \\
\text { A, ASGHAR, } \\
\text { KARIMY } \\
2011\end{array}$ & $\begin{array}{c}\text { ESTRADA, } \\
\text { GARCÍA, } \\
\text { SÁNCHEZ } \\
2009\end{array}$ & $\begin{array}{c}\text { MESSAH } \\
2011\end{array}$ & $\begin{array}{c}\text { GUERRAS } \\
2004\end{array}$ \\
\hline 1 & $\begin{array}{l}\text { Estructura } \\
\text { organizacional }\end{array}$ & $X$ & $X$ & $\mathrm{X}$ & & & $X$ \\
\hline 2 & $\begin{array}{l}\text { Dirección } \\
\text { Liderazgo }\end{array}$ & $y$ & & & $\mathrm{X}$ & & $\mathrm{X}$ \\
\hline 3 & $\begin{array}{l}\text { Recursos } \\
\text { humanos }\end{array}$ & $X$ & & & & & $X$ \\
\hline 4 & $\begin{array}{l}\text { Cultura } \\
\text { organizacional }\end{array}$ & $X$ & $X$ & & & $\mathrm{X}$ & $\mathrm{X}$ \\
\hline
\end{tabular}




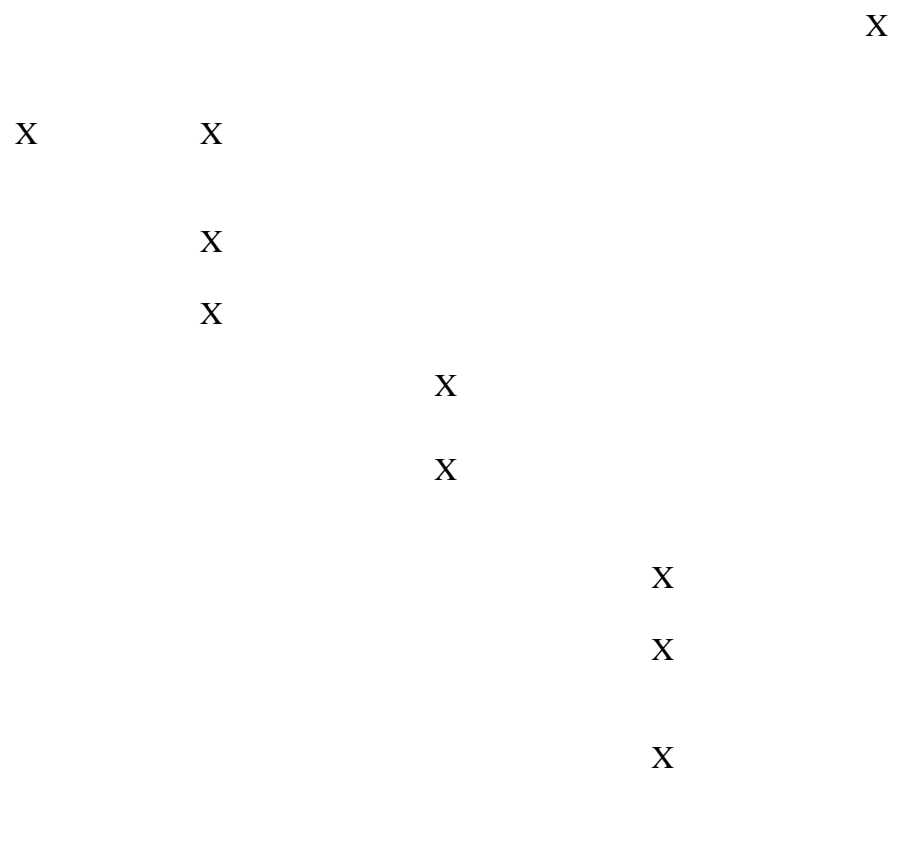

Fuente: Elaboración propia

A partir de los factores, se ubicaron las dimensiones más sobresalientes, que son: Visión, Liderazgo, Comunicación efectiva y otros factores. La estrategia de recopilación de datos para el análisis fue a través de un cuestionario validado a través de una muestra de la población conformada por empresarios y directivos que participan de forma directa e indirecta en la Confederación Patronal de la República Mexicana, concretamente en el Centro Empresarial de Ciudad Juárez. Este fue aplicado de manera directa en las sesiones de planeación al momento del registro de asistencia, supervisado directamente y asegurando que el instrumento haya sido contestado por los empresarios y directivos de las empresas. El número de empresarios y directivos que participan de manera regular en los procesos de planeación del Centro Empresarial COPARMEX Juárez; es decir, el marco muestral son 108, por lo tanto, la muestra que se calculó fue de 85 y se obtuvieron 83 respuesta, lo que refleja una tasa de respuesta del $97 \%$.

El presente artículo es descriptivo, relacional, exploratorio de corte transversal. Se analizaron los factores que afectan la IPE y la asociación de variables, que forma parte del análisis no paramétrico. Se determinaron las dimensiones para la pertinencia de las preguntas y/o variables de investigación que se seleccionaron para el análisis de datos. Las dimensiones determinadas son: visión, liderazgo y comunicación efectiva, empleando un enfoque asociativo entre variables mediante estadística descriptiva para analizar datos.

Para evaluar el nivel de confiabilidad del cuestionario se recurrió a la determinación de la consistencia interna, la cual busca determinar la 
proporción de la varianza en una escala (Oviedo y Campo, 2005). El coeficiente más utilizado es el Coeficiente Alfa de Cronbach (Oviedo y Campo, 2005), para evaluar el grado de confiabilidad de consistencia interna de una escala en que los ítems de un instrumento están correlacionados.

Los valores mínimos de consistencia interna del Coeficiente Alfa de Cronbach oscilan entre 0.70 a 0.90 . Debajo de este rango se considera inaceptable y por encima se presume la existencia de redundancia o duplicación (Hernández, Fernández y Baptista, 2006; Oviedo y Campo, 2005). Al aplicar este procedimiento se obtuvo un índice de 0.766 , lo cual significa un nivel aceptable. La variante para ítems dicotómicos es la técnica de Kuder Richardson (KR20) y para el caso del cálculo en SPSS el proceso es el mismo. Se presentan las variables en la siguiente dependencia tanto en el formato de dependientes e independientes de acuerdo con los objetivos específicos de la investigación (Ver Tabla 4).

Tabla 4. Operacionalización de variables

\section{Tipo de variable}

Independiente

Dependiente

Moduladora
Factores asociados a la falta de éxito. Implementación de la
Planeación Estratégica (IPE).

Empresarios y Directivos del centro empresarial.

\section{Descripción}

Se refiere a los factores que dificultad la IPE (Zaribaf, Hamid, 2014; Estrada, García, Sánchez, 2009; Guerras, 2004).

Se refiere a las variables que propician la IPE (Zaribaf, Hamid, 2014; Hasan Pour, 2001; Messah, 2011; Guerras, 2004).

Participantes directos e indirectos del Centro Empresarial COPARMEX Juárez que asciende a 108 empresarios o directivos

Fuente: Elaboración propia

Los datos fueron capturados, procesados, ordenados y analizados en el paquete estadístico SPSS con la debida codificación de las variables y sus respectivos análisis. El análisis de los datos procesados del instrumento incluyó análisis descriptivos univariado y bivariado. Los resultados se expresaron con medidas de tendencia central y de dispersión y las hipótesis fueron contrastadas mediante estadísticos paramétricos y no paramétricos. Se realizó un análisis de asociación entre las variables, pero para determinarlas fue necesario realizar el procedimiento factorial para agrupar los datos a partir de un conjunto de datos. 
El objetivo es buscar un número mínimo de dimensiones capaces de explicar el comportamiento y las relaciones de las variables (Seyed et. al. 2011). Las debilidades más sobresalientes detectadas son: visión, liderazgo, comunicación efectiva (socialización). Se utilizó el método aleatorio simple para seleccionar la unidad de muestra del listado de participantes, determinando una muestra de 85 encuestas.

El resultado de esta fase fue un instrumento de 15 reactivos que evalúan las cuatro dimensiones (visión, liderazgo, comunicación efectiva y otros factores) El cuestionario incluyó 20 preguntas, compuesto de 15 dicotómicas y cinco preguntas para recoger información general, de las cuales dos están relacionadas con el entrevistado y tres con la organización.

En la investigación participaron 83 empresarios y directivos que participan de manera regular en COPARMEX Juárez. El puesto de los entrevistados refleja que el $63 \%$ son directivos de las organizaciones empresariales consultadas, $19 \%$ accionistas y $16 \%$ jefes de departamento. La tipología de los entrevistados indica que el $33.7 \%$ cuenta con 3 años o menos de antigüedad laborando en la empresa, $21.7 \%$ entre 3 a 10 años, el $9.6 \%$ en el rango de 10 a 15 años. Para el caso de 15 años a 20 se indica un $15.6 \%$ y por último el 19.2\% más de 20 años en la organización. Para el caso de la antigüedad en el puesto, el 32.5\% cuenta con 3 años o menos, el $34.9 \%$ de 3 a 10 años, para el caso del rango de 10 a 15 años es de $9.6 \%$, de 15 a 20 años es del 15.6\% y por último más de 20 años en el puesto de la organización es del $19.2 \%$. El nivel académico de los entrevistados mostró que un $61 \%$ cuenta con licenciatura, 34\% con posgrado y el 5\% con una formación de media superior. Los participantes del género masculino en el estudio fueron del $76 \% \mathrm{y}$ del género femenino del $24 \%$.

\section{Hallazgos y conclusiones}

La investigación permitió analizar los factores detectados en base a la evidencia empírica en el contexto de las organizaciones empresariales aplicado en el Centro Empresarial COPARMEX Juárez. y se determinan las dimensiones sujetas de estudio: Visión: El enfoque de esta dimensión tiene que ver con la visión compartida institucional y directiva para el éxito y/o fracaso en la IPE. (Zaribaf \& Hamid, 2014; Estrada, García \& Sánchez, 2009; Guerras, 2004). Liderazgo: La línea de esta dimensión identificada tiene el enfoque de las organizaciones empresariales, el liderazgo juega un papel altamente relevante en la IPE (Zaribaf \& Hamid, 2014; Hasan Pour, 2011; Seyed, Reza, Asghar \& Karimy, 2011; Guerras, 2004). Comunicación efectiva: Se concreta en la comunicación de la visión en todas las jerarquías de la organización interna y externa para el éxito o fracaso de la IPE. (Zaribaf \& Hamid, 2014; Hasan Pour, 2011; Messah, 2011; Guerras, 2004). 
La pregunta general del estudio se enfocó en los factores asociados con el éxito o fracaso de la IPE que aparecen entre el planteamiento y su fase de implementación, resaltando que los factores que mayor éxito en el contexto de los empresarios y directivos del Centro Empresarial COPARMEX Juárez son la comunicación, visión y el ambiente externo.

Los supuestos específicos que se plantearon fueron: 1.-La falta de una visión compartida está asociada con el éxito o fracaso en la IPE. 2.-La falta de liderazgo está asociada con el éxito o fracaso en la IPE. 3.-La falta de comunicación efectiva de la visión futura está asociada con el éxito o fracaso en la IPE. Se comprobó que en el contexto del grupo de estudio, las variables anteriores están asociadas al éxito en la IPE. Para determinar el grado de asociación entre las variables, se utilizó el método de la correlación $R$ Pearson y para la contrastación de hipótesis se utilizó la misma prueba estadística. Se realizó un análisis para determinar la existencia de la asociación de las variables de estudio detectadas en base a la evidencia empírica (Liderazgo, Visión, Comunicación de la Visión y otros) (Ver Tabla 5).

Tabla 5. Correlación de factores de éxito o fracaso en la Implementación de la Planeación Estratégica (IPE).

$\begin{array}{llccccl}\text { No. } & \text { Factores } & \begin{array}{c}\text { R de } \\ \text { Pearson }\end{array} & \begin{array}{c}\text { Sig. P- } \\ \text { Valor }\end{array} & \begin{array}{c}\text { Muestra } \\ (\mathbf{n})\end{array} & \text { Interpretación } & \begin{array}{l}\text { Grado de } \\ \text { Asociación }\end{array} \\ 1 & \text { Liderazgo } & 0.23 & 0.04 & 83 & & \\ 2 & \text { Comunicación } & 0.6 & 0.0 & 83 & \text { Asociada } & \text { MEDIA } \\ 3 & \text { Visión } & 0.6 & 0.0 & 83 & \text { Asociada } & \text { MEDIA } \\ 4 & \text { Ambiente } & 0.5 & 0.0 & 83 & \text { Asociada } & \text { MEDIA } \\ & \begin{array}{l}\text { Externo } \\ \text { Estructura }\end{array} & 0.24 & 0.03 & 83 & & \\ & \text { organizativa } & & & & & \end{array}$

Fuente: Elaboración propia

La evidencia mostró que el factor liderazgo en el grupo de estudio no fue suficiente para concluir que está asociada al éxito de la implementación de la planeación en esta muestra sujeta de El $R$ de Pearson demuestra una asociación baja $(\mathrm{R}=0.23)$ el nivel de significancia sugiere que no es determinante $(\mathrm{p}<0.04)$. El estudio demuestra que el factor comunicación efectiva de la visión futura está asociada con el éxito o fracaso de la IPE. Un nivel de significancia del error del 5\%. Para el caso de la muestra sujeta de estudio la comunicación es un factor determinante para el éxito o fracaso de la IPE. El grado de asociación de $R$ de Pearson de los factores antes descritos concluye que la visión compartida está asociada con el éxito o fracaso de la IPE, con un nivel de significancia del error del $5 \%$.

Para el sector empresarial, contar con una visión estratégica que permita detonar proyectos, planes y expansión de negocios es clave para el éxito en la IPE en las organizaciones empresariales. Resalta de manera importante que el factor de comunicación tiene un grado de asociación con el 
éxito o fracaso en el grupo de estudio. Los modelos de planeación no consideran la socialización como mecanismos de comunicación de la implementación, por lo que se sugiere incluirlo en un modelo de adecuación para mejorar los procesos de IPE de las organizaciones. Para la conciliación de intereses de los grupos y construcción de una visión compartida para la IPE, la comunicación efectiva es altamente relevante en los resultados de IPE. El estudio determina nuevos hallazgos en el éxito de la IPE. El ambiente externo y estructura organizativa son factores para considerar en el éxito en la IPE. El factor ambiente externo está asociada con el éxito o fracaso de la IPE. Con un nivel de significancia del error del 5\%.

En el factor de estructura organizativa, la evidencia que se recaba no fue suficiente para concluir que el liderazgo está asociada al éxito de la implementación de la planeación debido a que a pesar de que el $R$ de Pearson de esta variable demuestra una asociación baja $(\mathrm{R}=0.24)$, el nivel de significancia sugiere que no es determinante $(\mathrm{p}<0.03)$. Bajo el contexto de inseguridad de este grupo de empresarios situados en el centro empresarial COPARMEX Juárez, México, el factor relevante para el éxito o fracaso de la IPE en primer término es el ambiente externo que muestra un grado de asociación mayor que el liderazgo.

Lo anterior puede explicar que las decisiones empresariales afectadas por la inseguridad tienen mayor influencia y afecta de manera directa en los modelos de planeación, a su vez genera la pertinencia a una adecuación de las metodologías de implementación hasta ahora utilizados. En base a lo anterior puede afirmar que bajo el contexto de estudio del grupo de empresarios y directivos del Centro Empresarial COPARMEX Juárez, los factores con mayores grados de asociación para el éxito en la IPE son comunicación, visión y ambiente externo. Parta el caso de los factores con menor grado de asociación son liderazgo y estructura organizativa. Bajo el contexto de este grupo de empresarios situados en el Centro Empresarial COPARMEX Juárez, las variables relevantes para el éxito de la IPE se encuadran en base a su contexto de retos del sector empresarial afectados por el entorno fronterizo y de seguridad, ya que la variable "ambiente externo" resulta más relevante por encima del liderazgo.

\section{References:}

1. Chinchilla, N. (1991). Teoría de la Organización. Contenido del Campo General: Status. Questions. Documento de Investigación. Universidad de Navarra. No. 218.

2. Conrado, A. C. (2000). La Necesidad de la Planeación Estratégica en las Organizaciones Industriales Modernas. Temas de Ciencia y Tecnología No. 11. Vol. 4.:17-28. 
3. De la Rosa, A. A. (2002). Teoría de la Organización y Nuevo Institucionalismo en el Análisis Organizacional. Universidad Autónoma Metropolitana (UAM). Administración y Organizaciones. No. 8. Vol. 4.: 13-44.

4. Deloitte \& Stracienta. (2007). Madurez en la Planeación Estratégica de las Empresas en México. Notimex El Universal. Recuperado: http://www.eluniversal.com.mx/notas/457056.html.

5. Di maggio, P. J. \& Powell, W. W. (1991). El Nuevo Institucionalismo en el Análisis Organizacional. Fondo de Cultura Económica. Universidad Autónoma Metropolitana (UAM).

6. Escobar, P. B \& Lobo, G. A. (2000). Aportaciones de la Teoría Contingente al Estudio de los Sistemas en las Organizaciones: Un Metanálisis. Departamento de Contabilidad y Economía Financiera. Universidad de Sevilla.

7. Estrada, B. R., García, P. D \& Sánchez, V. G. (2009). Barreras que dificultan la implementación estratégica en la pyme: una evidencia empírica en empresas mexicanas. Universidad Autónoma del Estado de Hidalgo, México.

8. Guerras, L. Á. (2004). Problemas Organizativos en el Proceso de la Dirección Estratégica. Universia Business Review. No. 1: 116-126.

9. Hasan Pour, E. (2011). Strategy Execution Capability: Case Study of Kebanoo Company. Faculty of Business Management. Islamic Azad University (IAU).

10. Hernández, S. R., Fernández, C. C. \& Baptista, L. P. (2006). Metodología de la Investigación. MCGRAW-HILL Interamericana.

11. Kotter, J. P. (1996). Leading Change. Harvard Business Review Press.

12. Messah, O. B. (2011). Factors Affecting the Implementation of Strategic Plans in Goverment Tertiary Institutions: A Survey of Selected Technical Training Institutes. European Journal of Business and Management. Kenya Methodist University. No. 53 Vol. 3: 85-105.

13. Moreno, L. Á. (2014). La Gestión de Talentos como Ventaja Competitiva Sostenible: Concordancia entre Percepciones y Actitudes de Directivos a Cargo de Recursos Humanos y Producción. Tesis Doctoral en Ciencias de la Administración. Universidad Autónoma de México (UNAM).

14. Okari, H., Letting, N, Gachunga. H. \& Katula, B. (2013). Influence of Organization Structure on Diversification Strategy and Performance of an Organization. Prime Journal of Business Administration Management. Vol.3.

15. Oviedo, H. C. \& Campo, A. A. (2005). Aproximación al Uso del Coeficiente Alfa de Cronbach. Revista Colombiana de Psiquiatría. Asociación Colombiana de Psiquiatría. Vol. XXXIV. No. 4: 572-580 
16. Porter, M. (1980). Estrategia Competitiva: Técnicas para el análisis de la empresa y sus competidores. Continental. Ed. 17.

17. Ramírez, G., Vargas, G. \& De la Rosa, A. (2001). Estudios Organizacionales y Administración. Contrastes y complementariedades: caminando hacia el eslabón perdido. №. 3: 753

18. Rivas, L. A. (2007). Mapas de las Teorías de la Organización: Una Orientación para empresas. Gaceta Ideas, CONCYTEG. Año. 2. No. 3: 35-67.

19. Robles, V. (2014). Una Introducción al Nuevo Institucionalismo Empresarial. Revista de Economía. Instituto Tecnológico de Roque. Celaya Guanajuato. Vol.1. No. 1: 31-40.

20. Scott, R. (1995), Institutions and Organizations. Ideas, Interests and Identities: Paperback: 360 pages Publisher: Sage (1995) Language: English ISBN: 978-142242224.M@n@gement, vol. 17(2), 136-140. doi:10.3917/mana.172.0136.

21. Seyed, N., Reza, M., Asghar, A. \& Karimy, D. (2011). Why does plans implementation falls? A study in the health service sector of Iran. A Journal of Business Management. Vol. 5 (23): 9831-9837

22. Zaribaf, M. \& Hamid, B. (2014). An Effective Factors Pattern Affecting Implementation of Strategic Plans. Bayrami Hamid Islamic Azad University, Firoozkuh Branch. Recuperado: http://www.indabook.org/d/Mehdi-Zaribaf-PhD-Shahrake-omidTehran-Iran-Bayrami.pdf 